\title{
Regional gastric emptying and gastric contractile activity in dyspepsia: new approach optimizing the
} consensus protocol

\author{
Ietsugu $^{a}$ M.V., Américo ${ }^{b}$ F.M., Pinto ${ }^{a}$ L.A., Próspero ${ }^{a}$ A.G., \\ Soares $^{1}$ G.A., Koga ${ }^{c}$ K.H., Miranda ${ }^{1}$ J.R.A.
}

${ }^{a}$ Department of Biophysics and Pharmacology, Bioscience Institute of Botucatu, 18618-000, São Paulo State University, Botucatu, São Paulo, Brazil.

bInstitute of Biological Sciences and Health, Mato Grosso Federal University, 78601-014 Barra do Garças, Mato Grosso, Brazil.

'Department of Tropical Diseases and Imaging Diagnosis, Botucatu Medical School, 18618000, São Paulo State University, Botucatu, São Paulo, Brazil.

jose.r.miranda@unesp.br 


\begin{abstract}
Consensus protocol organizes gastric emptying scintigraphy (GES). There is low adherence to the protocol, and its applicability is reduced due to poor connection between retention and functional disorders. To optimize the consensus protocol, we added a dynamic acquisition and other approaches for healthy and dyspeptic subjects. 14 patients with functional dyspepsia (FD) and 20 healthy volunteers were undergone GES. The dynamic acquisition was added to the activity versus time curve composed of 4-time points preconized. A dynamic set of 3060 images $(1 \mathrm{~Hz})$ were acquired between immediate and $60 \mathrm{~min}$ static images. Regions of interest were drawn to divide the stomach internally, and two gastric segmentations (similar areas and incisura) were adopted for all quantification of global GE and contractile parameters. Gastric retention at 4-time points preconized was not enough to identify differences between FD and healthy volunteers $(R=0.99 ; p<0.0009)$. Retention percentage of test meal in the proximal stomach employing segmentation, T_(1/2), MGET and lag phase analysis were higher in dyspeptic compared to healthy volunteers. Impaired intragastric meal distribution was observed in FD by similar areas $(p<0.02)$ and incisura $(p<0.001)$. Reduction in the amplitude of contraction from the proximal region ( $p<0.015$ and $p<0.003$ ) was observed in FD segmentation by similar areas and incisura, respectively. Impaired proximal stomach was observed in functional dyspepsia due to the insertion of dynamic acquisition proposed by consensus. Our approach can strengthen the consensus protocol and makes it capable of recognize and classify some diseases.
\end{abstract}

Keywords: gastric emptying scintigraphy, intragastric meal distribution, gastric contraction, dyspepsia, segmentation 


\section{INTRODUCTION}

Gastric emptying (GE) analysis is an important tool to assess gastric motility in clinical practice. Several methods have been used for this purpose [1], including gastric emptying scintigraphy (GES), considered the gold standard technique. However, due to a lack of standardization in data acquisition and quantification parameters, it is difficult to establish standard protocols. A consensus protocol was organized to define patient preparation, meal composition, acquisition protocols, and data processing $[2,3]$. The guideline consensus represents an essential tool to GES standardization, providing conditions to normalize reference patterns and compare nuclear medicine laboratory results.

The guideline consensus recommended static image acquisition at least the four time points: immediately and at 60, 120, and 240 minutes after meal ingestion. Furthermore, gastric meal retention in each moment should be calculated as a percentage of the initial meal retention [2]. Unfortunately, a recent study demonstrated low instruction compliance, although efforts had been made to increase the credibility of GES [3]. There are several disagreements between services and guidelines related to patient preparation and image processing. For instance, GE analysis is still calculated by gastric emptying half-time $\left(\mathrm{T}_{-}(1 / 2)\right)$ in detriment to gastric meal retention [3] and the selection of time points for GE studies by scintigraphy is also controversial [4-7]. Also, the lag time was not standardized to the meal proposed, either its measurement methods.

Moreover, a limitation of the guideline consensus is that GE retention values were obtained in normal subjects, and little is known about gastric motility in functional disorders. Functional dyspepsia (FD) is a usual gastrointestinal dysfunction characterized by nausea, vomiting, postprandial fullness, early satiety, and epigastric pain without organic disease that justifies the symptomatology [8, 9]. Roma III system classified FD into epigastric pain and postprandial distress [10], wherein visceral hypersensitivity and motility abnormalities are probably involved in disease etiology, respectively [11]. Dyspeptic patients usually had normal global GE $[12,13]$. Thus, the GE retention parameter isolated is not a reliable tool to explain symptoms [3, 13] because it does not reflect the organ function altogether [14]. 
However, the intragastric meal distribution (IMD) seems altered in dyspeptic patients, characterized by impaired gastric accommodation and consequent increase of meal in the proximal stomach $[11,15]$. Moreover, quantitative measurements of gastric contractility from proximal and distal regions and IMD might provide relevant information about gastrointestinal motility disorders and were suggested by previous studies $[2,13,16]$. To assess region gastric retention, regions of interest (ROI) are used [17] to segment the organ in proximal and distal areas. The fundus is functionally the proximal portion of the stomach, responsible for the receptive relaxation and accommodation, and then generates a pattern of tonic contractility around $1 \mathrm{cpm}$ (cycles per minute) $[18,19]$. The stomach's distal area is the antrum, responsible for generating phasic motor activity of $3 \mathrm{cpm}$ to grind the solid food and enable the GE [20]. However, there is no standardization to gastric division, and the adopted segmentation may influence the results [19].

The association among classical parameters such as global GE, IMD, and gastric contractility activity may help classify and recognize FD patients, corroborate abnormal physiology, and explain dyspeptic symptoms, especially when GE values are considered normal [2]. We aimed to compare the GES of healthy volunteers and FD patients to determine the optimized parameters to distinct them in clinical practice.

\section{MATERIALS AND METHODS}

\subsection{Subjects}

Fourteen patients with FD (4 men and 10 women; mean age $45.9 \pm 9.3$ years; range 25-59 years; mean body mass index [BMI]: $27.7 \pm 4.1 \mathrm{~kg} / \mathrm{m} 2$; range 22.0-35.1 kg/m2) selected from local university hospital according to Roma III system [10] and twenty healthy asymptomatic volunteers ( 5 men and 15 women; mean age $26.8 \pm 8.26$ years; range 15-51 years; mean BMI: $24.2 \pm 3.2$ $\mathrm{kg} / \mathrm{m} 2$; range $20.0-31.4 \mathrm{~kg} / \mathrm{m} 2$ ) with no history of gastrointestinal disease or abdominal surgery underwent to GES. Patients on medications were oriented to discontinue narcotics, prokinetic, opiate analgesic, anticholinergic, and antispasmodic agents at least two days before the exam. All women were evaluated during the first ten days of menstrual cycle [2]. The investigation was 
performed according to the Declaration of Helsinki, and the local Ethics Committee previously approved the study protocol (Statement 129/2012). All subjects signed informed consent.

\subsection{Gastric Scintigraphy Imaging Protocols}

After an overnight fast, participants ingested a meal composed of a sandwich and $120 \mathrm{ml}$ of water within $10 \mathrm{~min}$. The sandwich consisted of two slices of white bread slices, strawberry jelly, two eggs white scrambled radiolabeled with $37 \mathrm{MBq} 99 \mathrm{mTc}$ sulfur-colloid. The meal's caloric value was $255 \mathrm{kcal}$, containing $72 \%$ carbohydrates, $24 \%$ protein, $2 \%$ fat, and $2 \%$ fibers [2].

Immediately after ingestion, participants were instructed to stand upright between the dual-head gamma camera (Sopha Vision DST, Sopha Medical Vision America, Twinsburg, OH, USA) equipped with low energy all-purpose collimators. Images were acquired using $140 \mathrm{keV} 99 \mathrm{mTc}$ photopeak, 20\% window, and acquisition matrix size $64 \times 64$ and $128 \times 128$ to dynamics and statics images, respectively. Static images were obtained, during 1 min time, immediately and at 60, 120, and $240 \mathrm{~min}$ after test meal ingestion [2]. A dynamic set of 3060 images in a sampling frequency of $1 \mathrm{~Hz}$ were acquired between immediate and $60 \mathrm{~min}$ static images. All images were acquired in anterior and posterior views.

\subsection{Image Processing}

Data were analyzed using MatLab (Mathworks Inc., Natick, MA, USA) and Origin (Origin Lab, USA). All anterior and posterior images were corrected to radionuclide decay and attenuation, resulting in geometric mean images (GMI). The matrix of static GMI was resized to $64 \times 64$ to be the same dimension of dynamic GMI. All 3060 dynamics GMI were summed to result in a single 51 min image, and a ROI was manually drawn around the whole stomach. ROI from the entire stomach was segmented internally, providing proximal and distal regions. Segmentation was done using two methodologies : according to similar areas established by a perpendicular line at the midpoint of the highest longitudinal axis of the organ [15, 21-23] and in two different sizes delimited by gastric incisura [19, 24, 25] (Fig. 1). ROIs were copied on 0 (immediately after test meal ingestion) and at 60, 120, and 240 min static GMI. 

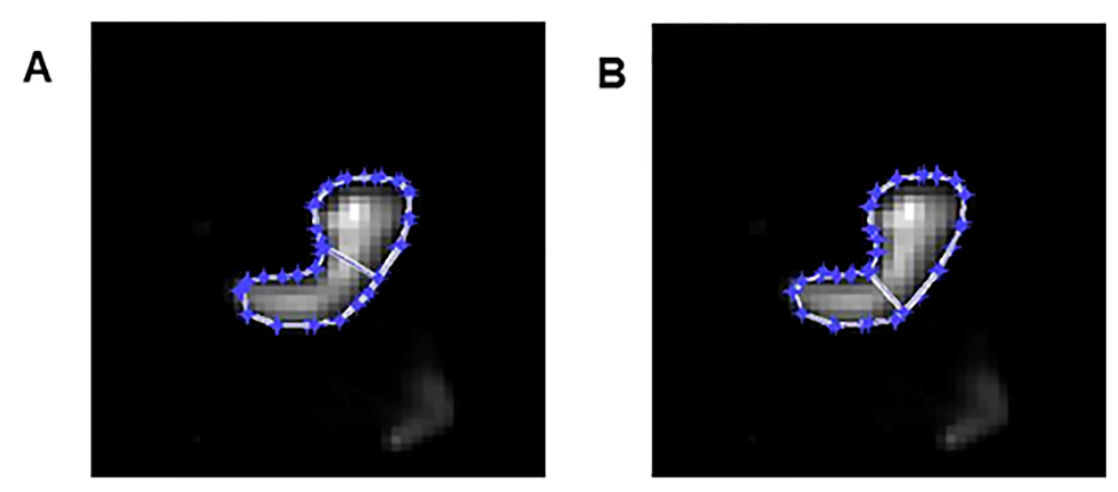

Fig. 1. Gastric segmentations by similar areas (A) and gastric incisura (B).

Activity versus time curves from the whole stomach, proximal and distal regions, was composed by counts from all static GMI and by dynamic images transformed into static (the sum of the images every 60 frames - 1 minute, corresponds to a static image). These curves were used to assess global GE, lag phase, and IMD, while isolated dynamic acquisition was applied to evaluate proximal and distal gastric contractile activity.

Data Analysis

The following parameters evaluated global GE: (a) Gastric emptying half-time (T_(1/2)) expressed as the time (min) when initial activity decreased 50\% [2]; (b) Mean gastric emptying time (MGET) (min) [26], calculated by statistical moments through pondered temporal average normalized by the area under the curve (AUC) of total stomach [27]; (c) Retention values at 60, 120 and 240 min quantified as the percentage of maximum activity in the image immediately after test meal ingestion $[2,15,22]$. The retention times at intermediate moments were calculated by adding 60 seconds of dynamic images at each time (10,20,30 and 40 minutes). The lag phase was also calculated by two distinct methods : time (min), in which the initial activity decreased 10\% [28] and time (min) which the highest activity value in the distal region was verified [29], which coincides with the inflection point of total stomach curve [21] and with the emergence of the test meal in the duodenum [2].

The IMD quantification was performed by the ratio between AUC of proximal (AUCprox) and distal stomach (AUCdist) $[15,19,22]$. Spectral analyses from proximal and distal regions were 
performed using Fast Fourier Transform (FFT) to verify the dominant frequency in both segmentations, defined as the highest power spectrum observed in each gastric region. The signals were filtered by a bi-directional Butterworth band-pass [19, 29, 30] with a cutoff frequency at 5-120 $\mathrm{mHz}$ to assess proximal and distal regions. The amplitude of gastric contractions was estimated by AUC [18] for proximal and distal regions.

Data were tested to normal distribution by Shapiro-Wilk's test. All data were represented by mean \pm SD. Student's t-test was performed to compare FD and healthy volunteer's data, and paired t-test was applied to confront segmentations. Pearson correlation was used to evaluate gastric retention curves. Differences were considered significant for values of $\mathrm{p}<0.05$.

\section{RESULTS AND DISCUSSION}

The percentage of gastric retention at $60(46.15 \pm 14.58 \%$ vs $49.60 \% \pm 9.78 \%), 120(14.80 \% \pm$ $10.48 \%$ vs $18.08 \% \pm 9.54 \%)$ and $240 \min (1.24 \% \pm 2.16 \%$ vs $1.29 \% \pm 3.18 \%)$ proposed by GES consensus were not significantly different among healthy volunteers and dyspeptic patients $\left(\mathrm{R}^{2}=\right.$ 0.99) (Fig. 2).

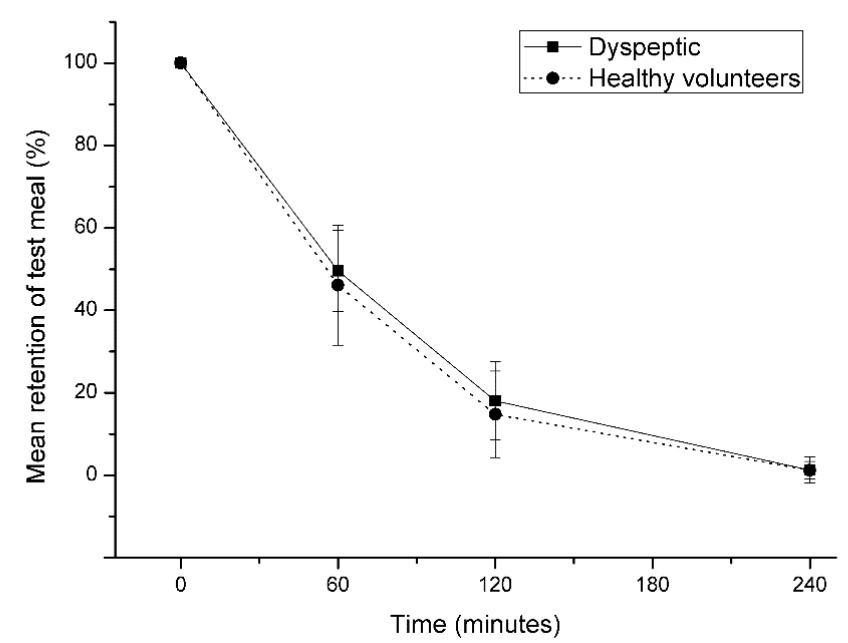

Figure 2: Gastric retention of meal test in 4-time points proposed by consensus protocol for healthy volunteers and dyspeptic patients. regular screen monitor. Nevertheless, be aware that the higher the resolution, the larger the final file. 
In contrast, increased meal retention was found for the FD patients by T_ $(1 / 2)(\mathrm{p}<0.02)$ and MGET $(\mathrm{p}<0.05)$ quantification. Furthermore, dyspeptic patients also presented a longer lag phase for two methods of quantification: initial activity decreased by $10 \%(\mathrm{p}<0.05)$ and incisura segmentation $(\mathrm{p}<0.03)$. Although, the lag phase difference between groups was not significant in gastric segmentation by similar areas. In the segmentation comparison, we found a larger delay time $(p<0.04)$ of GE in the FD when applied the segmentation by incisura (Table 1).

In our study, a 60 min dynamic acquisition sequence was successfully introduced among the static acquisitions proposed by consensus protocol and other quantifications approaches were employed. This addition does not increase the patient's exposure to ionizing radiation and optimize their time standing in the nuclear medicine service. Furthermore, our approach allowed exploring important gastrointestinal motility features and clarifying differences between healthy subjects and dyspeptic patients, which was not addressed in previous studies using a similar methodology [16].

Table 1: Gastric emptying parameters (min) of healthy volunteers and dyspeptic patients.

\begin{tabular}{cccc}
\hline Gastric emptying parameters $(\min )$ & Healthy volunteers & $\begin{array}{c}\text { Dyspeptic } \\
\text { patients }\end{array}$ & $\mathbf{P}$ \\
\hline $\mathrm{T}_{1 / 2}$ & $54.90 \pm 10.90$ & $64.39 \pm 11.28$ & $<0.02$ \\
$\mathrm{MGET}$ & $48.49 \pm 8.23$ & $55.02 \pm 9.27$ & $<0.05$ \\
Lag phase $-10 \% \mathrm{GE}$ & $17.14 \pm 5.39$ & $22.54 \pm 7.48$ & $<0.05$ \\
Lag phase - Segmentation by similar areas & $27.71 \pm 16.32$ & $34.94 \pm 16.61$ & 0.19 \\
Lag phase - Segmentation by gastric incisura & $29.88 \pm 16.56$ & $46.96 \pm 25.03^{*}$ & $<0.03$ \\
\hline
\end{tabular}

Data are expressed as Mean \pm SD. MGET: Mean Gastric Emptying Time; GE: Gastric Emptying. $* \mathrm{p}<0.04$ for comparison between segmentations.

In this context, retention values at 60, 120, and 240 minutes should be carefully considered since many dyspeptic patients presented a normal GES [31, 32]. In the results obtained using consensus protocol, it was impossible to identify differences in retention between healthy and FD patients $(\mathrm{R}=0.99)$ (Fig. 2). However, using our approach, both T_(1/2) and MGET were higher in dyspeptic patients than in normal subjects $(\mathrm{p}<0.02$ and $\mathrm{p}<0.05$, respectively). Also, the lag phase 
was increased in FD for $10 \%$ test meal emptying $(\mathrm{p}<0.05)$ and for the highest activity in the distal region by incisura segmentation $(\mathrm{p}<0.03)$ due to the greater proximal area.

Lag phase and $\mathrm{T}_{1 / 2}$ are classical parameters to quantify GE [3] despite considerable disadvantages such as quantification in time activity curves with irregular shapes, inability to provide regional GE information, and mensuration applying only 4-time points on the curve [27]. Thus, our study also quantified MGET to get information about the entire process of GE. Regardless of the methodology employed, there was a considerable intra-individual variation related to GE parameters of quantification, previously corroborated by Camilleri et al. [33]. It is appropriate to reinforce that none of the analysis tool isolated seems reasonable to represent gastric function after the meal.

The curve profile of the mean retention time of test meal for both groups and segmentation is shown in Fig. 3. The similar area segmentation (Fig. 3A) showed that FD patients presented increased meal retention in the proximal stomach compared to healthy volunteers during the entire measurement. The same profile was observed for the incisura segmentation (Fig. 3B), where higher retention values were found in dyspeptic patients' proximal stomach. The quantification of IMD in terms of the ratio between $\mathrm{AUC}_{\text {prox }}$ and $\mathrm{AUC}_{\text {dist }}$ is shown in Fig. 4. Despite the segmentation method adopted, the proximal region retention was higher on FD patients than healthy volunteers. Furthermore, the incisura segmentation resulted in higher proximal region retention than similar areas segmentation for both groups. 
A

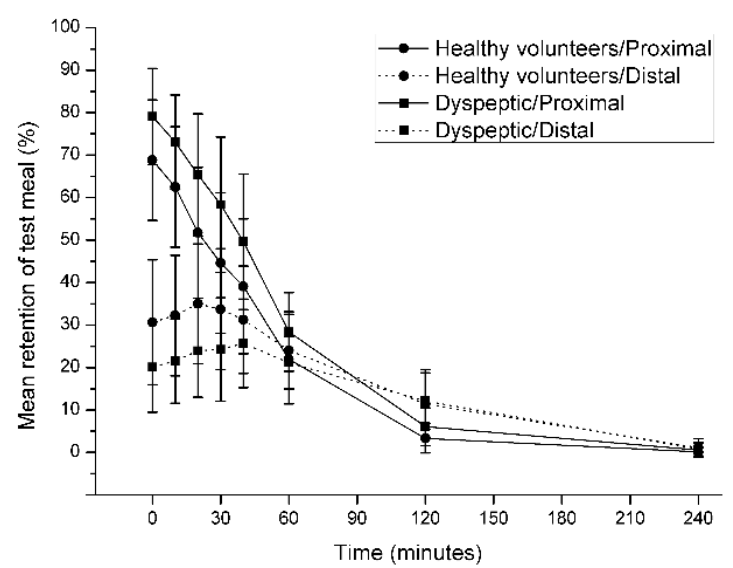

B

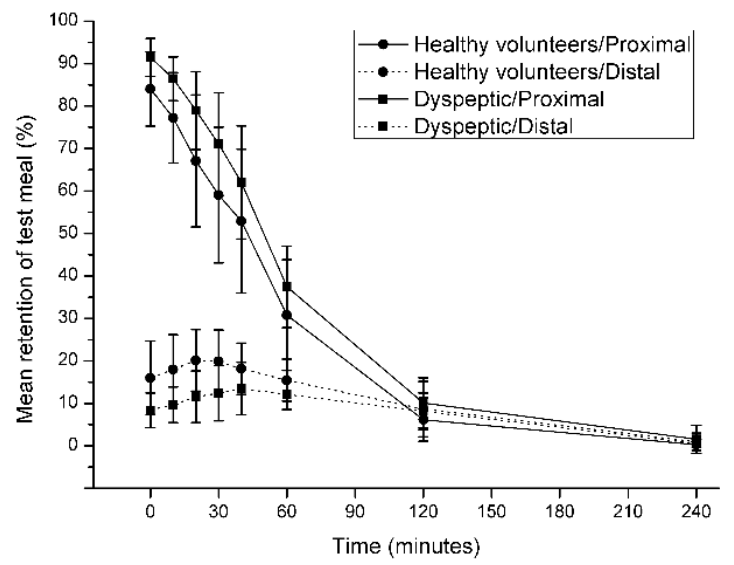

Figure 3 : Mean retention time of test meal (\%) versus time curves from proximal and distal regions of healthy volunteers and dyspeptic patients obtained by (A) similar areas and (B) incisura segmentation.

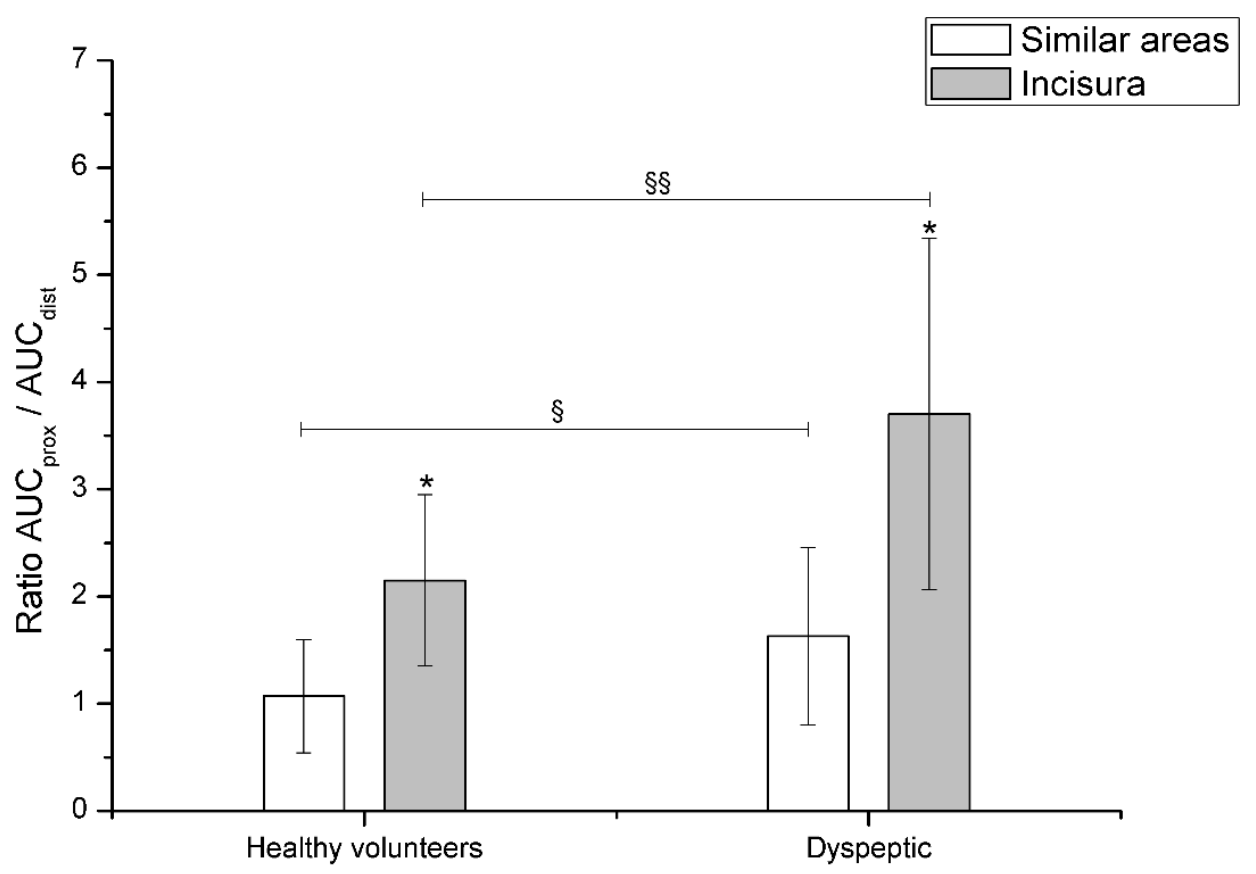

Figure 4: Intragastric meal distribution obtained by the ratio AUCprox/AUCdist for healthy volunteers and dyspeptic patients. Data is shown as mean $\pm \mathrm{SD}$. $* \mathrm{p}<0.05$ and $* * \mathrm{p}<0.001$ between incisura and similar areas segmentation. $\S p<0.02$ between healthy volunteers and dyspeptic patients for similar areas segmentation; $\S \S p<0.001$ between healthy volunteers and dyspeptic patients for gastric incisura segmentation. 
The goal of diagnosing delayed GE is to identify patients who will benefit from either a prokinetic drug or other treatment to relieve symptoms. For an accurate GES analysis is necessary to understand that multiple factors affect GE, particularly the separate roles of the fundus and antrum [13].

Our results demonstrate that proximal retention over time was, as expected, greater when the stomach was segmented according to gastric incisura due to a larger proximal area than segmentation by similar areas $(\mathrm{p}<0.001)$. Differences of IMD between healthy volunteers and dyspeptic individuals were perceived both in segmentation by similar areas and by incisura $(\mathrm{p}<0.02$ and $\mathrm{p}<0.001$, respectively) confirming proximal retention in FD (Fig. 3A-B). In addition, our study verified increased proximal retention in relation to the distal content, which was reiterated by higher average values of the ratio between AUCprox and AUCdist (Fig. 4), and these findings may be associated with early satiety in dyspeptic [34].

The dynamic acquisition sequence enabled the assessment of the proximal and distal gastric dominant frequency of contraction and amplitude (Table 2). A dominant frequency in the proximal region around $0.81 \mathrm{cpm}$ (similar to the standard of $1 \mathrm{cpm}$ ) was registered in healthy volunteers and dyspeptic individuals, independently from segmentation adopted. There were no significant differences between groups related to the well-known frequency from the distal region around 3 cpm, regardless of the segmentation adopted. A smaller amplitude of contraction in the proximal region was observed in dyspeptic patients compared to healthy volunteers in both segmentations. However, no difference was found in the amplitude from the gastric distal region for both groups.

Orthey et al. [16] provided reference values using a $20 \mathrm{~min}$ dynamic acquisition and the assessment of the GE in terms of IMD as a standard protocol. Our results are in accordance with the reference values, however, the proposed protocol was not applied in FD patients. The dominant frequency around $1 \mathrm{cpm}$ was not observed in this study, probably due to the filtering band used. Koskenpato et al. [21], Piessevaux et al. [22], and Gonlachanvit et al. [23] used solid meals to assess IMD and differences between FD and controls, but no significant differences were found; however, the test meal was different from that employed in consensus protocol and adopted in our study. 
Table 2: Dominant frequencies of regional gastric contractions (cpm) and amplitude of regional gastric contractions (counts vs. second) in healthy volunteers and dyspeptic patients according to gastric segmentation.

\begin{tabular}{|c|c|c|c|}
\hline $\begin{array}{c}\text { Gastric region - } \\
\text { segmentation approach }\end{array}$ & Healthy volunteers & $\begin{array}{l}\text { Dyspeptic } \\
\text { patients }\end{array}$ & $\mathbf{P}$ \\
\hline \multicolumn{4}{|l|}{ Frequency (cpm) } \\
\hline $\begin{array}{l}\text { Proximal region - similar } \\
\text { areas }\end{array}$ & $0.79 \pm 0.14$ & $0.82 \pm 0.16$ & 0.55 \\
\hline $\begin{array}{l}\text { Proximal region - } \\
\text { incisura }\end{array}$ & $0.82 \pm 0.18$ & $0.85 \pm 0.16$ & 0.68 \\
\hline $\begin{array}{l}\text { Distal region - similar } \\
\text { areas }\end{array}$ & $3.07 \pm 0.30$ & $2.90 \pm 0.38$ & 0.23 \\
\hline Distal region - incisura & $3.15 \pm 0.29$ & $3.02 \pm 0.33$ & 0.24 \\
\hline $\begin{array}{l}\text { Amplitude (counts vs. } \\
\text { second) }\end{array}$ & & & \\
\hline $\begin{array}{l}\text { Proximal region - similar } \\
\text { areas }\end{array}$ & $2121.82 \pm 1180.30$ & $1248.38 \pm 405.90$ & $<0.015$ \\
\hline $\begin{array}{l}\text { Proximal region - } \\
\text { incisura }\end{array}$ & $1824.70 \pm 712.79$ & $1060.46 \pm 340.33$ & $<0.003$ \\
\hline $\begin{array}{l}\text { Distal region - similar } \\
\text { areas }\end{array}$ & $1517.66 \pm 619.90$ & $1430.64 \pm 497.20$ & 0.69 \\
\hline Distal region - incisura & $1026.58 \pm 347.86$ & $875.50 \pm 416.88$ & 0.16 \\
\hline
\end{tabular}

Data are expressed as Mean \pm SD. Cpm: cycles per minute.

Dynamic scintigraphy has been used to assess antral motility [30, 35, 36], although little is known about the dominant frequency and amplitude of contractions related to gastric regions in dyspepsia. Due to this fact, Consensus also suggests the importance of gastric contraction analysis [2]. Our data demonstrate that the dominant frequency in the gastric regions was similar between 
gastric segmentation methods. Moreover, contraction frequencies in proximal and distal regions were not different between groups (Table 2). A dominant frequency around $0.81 \mathrm{cpm}$ was observed in the proximal region $[18,19]$, whereas the frequency did not differ from $3 \mathrm{cpm}$ in distal region $[18,19,30]$ (Table 2). Functional dyspepsia is mainly characterized by epigastric pain and fullness, which is related to gastric motility abnormalities [11]. However, according to our results, dominant frequency alterations in dyspeptic patients cannot explain fullness as a symptom in whatever gastric region. On the other hand, the amplitude of contractions in the proximal region was lower in FD than in healthy volunteers by similar areas $(\mathrm{p}<0.015)$ and by incisura $(\mathrm{p}<0.003)$ segmentations. Smaller amplitude contractions in the proximal region can be responsible for higher T_(1/2), MGET, lag phase, and IDM were altered in these patients. In addition, similar amplitudes in the distal region allow an understanding of the lack of global GE difference between groups.

Assessment of traditional and new methods such as MGET, T_(1/2), IDM, retention of the meal (\%) in each gastric region, and lag phase analysis during standard GES may yield additional information and better assess gastroparesis symptoms and abnormal gastric motility [16, 34]. It was possible to observe impaired IMD and a reduction in proximal gastric amplitudes in dyspepsia due to the insertion of dynamic acquisition between static images proposed by Consensus. Nowadays, few laboratories are fully compliant with protocol consensus, and changes that make its use wider can improve this rate. Only gastric retention obtained in 4-time points was not enough to identify differences between dyspeptic patients and healthy volunteers. A dynamic acquisition and a blended analysis were able to increase accuracy and adding information during routine solid-meal GES.

One of the difficulties of this procedure is keeping the patient at rest for this time. In this proposal, it was successfully achieved using a support para o patient remained seated with the chest vertical, supported by the gamma-chamber. 


\section{CONCLUSION}

A dynamic acquisition inserted during the static records proposed by the consensus expands the ability to differentiate dyspeptic patients and healthy volunteers and makes it possible to assess different important parameters (IMD, MGET, lag phase, retention, and frequency) in the clinical analysis of patients.

\section{ACKNOWLEDGMENT}

Acknowledgment to FAPESP, CAPES and CNPq.

\section{REFERENCES}

[1] Szarka LA and Camilleri M. Methods for measurement of gastric motility. American journal of physiology. Gastrointestinal and liver physiology 2009;296:G461-75.

[2] Abell TL, Camilleri M, Donohoe K, Hasler WL, Lin HC, Maurer AH, et al. Consensus recommendations for gastric emptying scintigraphy: a joint report of the American Neurogastroenterology and Motility Society and the Society of Nuclear Medicine. Journal of nuclear medicine technology 2008;36:44-54.

[3] Farrell MB, Costello M, McKee JD, Gordon LL, and Fig LM. Compliance with GastricEmptying Scintigraphy Guidelines: An Analysis of the Intersocietal Accreditation Commission Database. Journal of nuclear medicine technology 2017;45:6-13.

[4] Klingensmith WC, 3rd and Lawrence SP. The gastric emptying study: protocol design considerations. Journal of nuclear medicine technology 2008;36:195-9.

[5] Zinsmeister AR, Bharucha AE, and Camilleri M. Comparison of calculations to estimate gastric emptying half-time of solids in humans. Neurogastroenterology and motility : the official journal of the European Gastrointestinal Motility Society 2012;24:1142-5.

[6] Pathikonda M, Sachdeva P, Malhotra N, Fisher RS, Maurer AH, and Parkman HP. Gastric emptying scintigraphy: is four hours necessary? Journal of clinical gastroenterology 2012;46:20915. 
[7] Vasavid P, Chaiwatanarat T, Pusuwan P, Sritara C, Roysri K, Namwongprom S, et al. Normal Solid Gastric Emptying Values Measured by Scintigraphy Using Asian-style Meal:A Multicenter Study in Healthy Volunteers. Journal of neurogastroenterology and motility 2014;20:371-8.

[8] Manabe N and Haruma K. Diagnosis and treatment of dyspeptic patients in Japan. Journal of gastroenterology and hepatology 2011;26 Suppl 3:27-31.

[9] Lee IS, Wang H, Chae Y, Preissl H, and Enck P. Functional neuroimaging studies in functional dyspepsia patients: a systematic review. Neurogastroenterology and motility : the official journal of the European Gastrointestinal Motility Society 2016;28:793-805.

[10] Drossman DA. Rome III: the new criteria. Chinese journal of digestive diseases 2006;7:181-5.

[11] Miwa H, Watari J, Fukui H, Oshima T, Tomita T, Sakurai J, et al. Current understanding of pathogenesis of functional dyspepsia. Journal of gastroenterology and hepatology 2011;26 Suppl $3: 53-60$.

[12] Maurer AH and Parkman HP. Update on gastrointestinal scintigraphy. Seminars in nuclear medicine 2006;36:110-8.

[13] Maurer AH. Gastrointestinal Motility, Part 1: Esophageal Transit and Gastric Emptying. Journal of nuclear medicine technology 2016;44:1-11.

[14] Troncon LE, Bennett RJ, Ahluwalia NK, and Thompson DG. Abnormal intragastric distribution of food during gastric emptying in functional dyspepsia patients. Gut 1994;35:327-32.

[15] Troncon LE, Herculano JR, Jr., Savoldelli RD, Moraes ER, Secaf M, and Oliveira RB. Relationships between intragastric food maldistribution, disturbances of antral contractility, and symptoms in functional dyspepsia. Digestive diseases and sciences 2006;51:517-26.

[16] Orthey P, Dadparvar S, Parkman HP, and Maurer AH. Enhanced Gastric Emptying Scintigraphy to Assess Fundic Accommodation Using Intragastric Meal Distribution and Antral Contractility. Journal of nuclear medicine technology 2019;47:138-43.

[17] Madsen JL. Scintigraphic assessment of gastrointestinal motility: a brief review of techniques and data interpretation. Clinical physiology and functional imaging 2014;34:243-53.

[18] Americo MF, Oliveira RB, Romeiro FG, Baffa O, Cora LA, and Miranda JR. Scintigraphic validation of AC Biosusceptometry to study the gastric motor activity and the intragastric 
distribution of food in humans. Neurogastroenterology and motility : the official journal of the European Gastrointestinal Motility Society 2007;19:804-11.

[19] Americo MF, Ietsugu MV, Romeiro FG, Cora LA, Oliveira RB, and Miranda JR. Effects of meal size and proximal-distal segmentation on gastric activity. World journal of gastroenterology : WJG 2010;16:5861-8.

[20] Tack J. Gastric motor disorders. Best practice \& research. Clinical gastroenterology 2007;21:633-44.

[21] Koskenpato J, Kairemo K, Korppi-Tommola T, and Farkkila M. Role of gastric emptying in functional dyspepsia: a scintigraphic study of 94 subjects. Digestive diseases and sciences 1998;43:1154-8.

[22] Piessevaux H, Tack J, Walrand S, Pauwels S, and Geubel A. Intragastric distribution of a standardized meal in health and functional dyspepsia: correlation with specific symptoms. Neurogastroenterology and motility : the official journal of the European Gastrointestinal Motility Society 2003;15:447-55.

[23] Gonlachanvit S, Maurer AH, Fisher RS, and Parkman HP. Regional gastric emptying abnormalities in functional dyspepsia and gastro-oesophageal reflux disease. Neurogastroenterology and motility : the official journal of the European Gastrointestinal Motility Society 2006;18:894904.

[24] van den Elzen BD, Bennink RJ, Holman R, Tytgat GN, and Boeckxstaens GE. Impaired drinking capacity in patients with functional dyspepsia: intragastric distribution and distal stomach volume. Neurogastroenterology and motility : the official journal of the European Gastrointestinal Motility Society 2007;19:968-76.

[25] Camilleri M, Breen M, Ryks M, and Burton D. Proximal and overall gastric emptying of solids in patients with reduced gastric volume accommodation compared to matched controls. Digestive diseases and sciences 2011;56:1729-34.

[26] Teixeira MC, Magalhaes I, Galvao PV, Souza GS, Miranda JR, Oliveira RB, et al. Assessment of gastrointestinal motility in renal transplant recipients by alternate current biosusceptometry. Transplantation proceedings 2012;44:2384-7. 
[27] Podczeck F, Newton JM, and Yuen KH. The description of the gastrointestinal transit of pellets assessed by gamma scintigraphy using statistical moments. Pharmaceutical research 1995;12:376-9.

[28] Odunsi ST and Camilleri M. Selected interventions in nuclear medicine: gastrointestinal motor functions. Seminars in nuclear medicine 2009;39:186-94.

[29] Urbain JL and Charkes ND. Recent advances in gastric emptying scintigraphy. Seminars in nuclear medicine 1995;25:318-25.

[30] Misiara GP, Troncon LE, Moraes ER, and Secaf M. Comparison between manual and automated techniques for assessment of data from dynamic antral scintigraphy. Annals of nuclear medicine 2008;22:761-7.

[31] Szarka LA and Camilleri M. Gastric emptying. Clinical gastroenterology and hepatology : the official clinical practice journal of the American Gastroenterological Association 2009;7:823-7.

[32] Maurer AH. Advancing gastric emptying studies: standardization and new parameters to assess gastric motility and function. Seminars in nuclear medicine 2012;42:101-12.

[33] Camilleri M, Iturrino J, Bharucha AE, Burton D, Shin A, Jeong ID, et al. Performance characteristics of scintigraphic measurement of gastric emptying of solids in healthy participants. Neurogastroenterology and motility : the official journal of the European Gastrointestinal Motility Society 2012;24:1076-e562.

[34] Orthey P, Yu D, Van Natta ML, Ramsey FV, Diaz JR, Bennett PA, et al. Intragastric Meal Distribution During Gastric Emptying Scintigraphy for Assessment of Fundic Accommodation: Correlation with Symptoms of Gastroparesis. Journal of nuclear medicine : official publication, Society of Nuclear Medicine 2018;59:691-7.

[35] Urbain JL, Vekemans MC, Parkman H, Van Cauteren J, Mayeur SM, Van den Maegdenbergh V, et al. Dynamic antral scintigraphy to characterize gastric antral motility in functional dyspepsia. Journal of nuclear medicine : official publication, Society of Nuclear Medicine 1995;36:1579-86.

[36] Misiara GP, Troncon LE, Hara SH, and Secaf M. Dynamic antral scintigraphy following solid and liquid meals in healthy human subjects. Nuclear medicine communications 2007;28:47983. 